\title{
Spatio-temporal Control of ERK Pulse Frequency Coordinates Fate Decisions during Mammary Acinar Morphogenesis
}

Pascal Ender ${ }^{1}$, Paolo Armando Gagliardi ${ }^{1}$, Maciej Dobrzyński ${ }^{1}$, Coralie Dessauges ${ }^{1}$, Thomas Höhener ${ }^{1}$, Marc-Antoine Jacques ${ }^{1}$, Andrew R. Cohen ${ }^{2}$ and Olivier Pertz $1 *$

${ }^{1}$ Institute of Cell Biology, University of Bern, Baltzerstrasse 4, 3012 Bern, Switzerland

2 Department of Electrical and Computer Engineering, Drexel University, 3120-40 Market Street, Suite 313, Philadelphia, PA 19104, USA

${ }^{*}$ Correspondence and requests for materials should be addressed to O.P. (email: olivier.pertz@izb.unibe.ch).

\begin{abstract}
The signaling events controlling proliferation, survival, and apoptosis during mammary epithelial acinar morphogenesis remain poorly characterized. By imaging single-cell ERK activity dynamics in MCF10A acini, we find that these fates depend on the frequency of ERK pulses. High pulse frequency is observed during initial acinus growth, correlating with rapid cell motility. Subsequent decrease in motility correlates with lower ERK pulse frequency and quiescence. Later, during lumen formation, coordinated ERK waves emerge across multiple cells of an acinus, correlating with high and low ERK pulse frequency in outer surviving and inner dying cells respectively. A PIK3CA H1047R mutation, commonly observed in breast cancer, increases ERK pulse frequency and inner cell survival, causing loss of lumen formation. Optogenetic entrainment of ERK pulses causally connects high ERK pulse frequency with inner cell survival. Thus, fate decisions during acinar morphogenesis are fine-tuned by different spatio-temporal coordination modalities of ERK pulse frequency.
\end{abstract}




\section{Introduction}

Development of the mammary gland involves formation of a rudimentary gland during embryogenesis, followed by proliferation and branching invasion led by multi-layered terminal end buds (TEBs) during puberty. Cells in the inner TEB layers then undergo apoptosis to form the ductal lumen. During pregnancy, secretory alveoli are then formed at the ends of the ductal tree (Inman et al., 2015; Paine and Lewis, 2017). Morphogenesis of this complex structure requires spatial and temporal control of cell fates such as proliferation, migration and death. However, the spatio-temporal signaling events that regulate such fate decisions remain poorly explored. The epidermal growth factor (EGF) receptor (EGFR) - extracellular regulated kinase (ERK) signaling cascade is a key signaling pathway involved in mammary gland development. EGFR-ERK signaling results in the upregulation of gene products involved in a wide variety of processes such as proliferation, survival, migration and differentiation (Lavoie et al., 2020). EGFR activity is required for mammary gland morphogenesis in mice (Sebastian et al., 1998). Paracrine amphiregulin release by the matrix metallo-protease ADAM-17 and its binding to EGFR mediates the effects of estrogen receptor $\alpha(E R \alpha)$ to promote mammary gland development and growth (Ciarloni et al., 2007; Sternlicht et al., 2005). EGFR-dependent ERK activity is enriched at the front of elongating tubes and coordinates cell migration (Huebner et al., 2016). In MCF10A acini, oncogenic ERK activation suppresses apoptosis and thus lumen formation (Reginato et al., 2005). In primary mammary cell culture, ERK activity is also crucial for survival (Finlay et al., 2000).

ERK activity dynamics rather than steady-states control fate decisions in mammary cells. TGFa-induced sustained ERK activation drives branching, whereas FGF7induced transient ERK activation stimulates proliferation in primary murine organoids (Fata et al., 2007). In non-tumorigenic MCF10A mammary epithelial cells cultured in 2D, fate decisions are controlled by the average frequency of asynchronous, singlecell ERK activity pulses of constant amplitude and duration, that we refer to as ERK frequency from now on. Low ERK frequency predisposes cells to apoptosis, whereas high ERK frequency drives survival and cell cycle progression (Albeck et al., 2013; Gagliardi et al., 2020). Single-cell ERK dynamics have, however, never been measured in 3D mammary acini. This can be performed by culturing single MCF10A cells in matrigel, that in approximately two weeks, yield hollow acini that retain key features of in vivo breast alveoli. For sake of clarity, and based on previous work 
(Anderson et al., 2010; Debnath et al., 2003), we subdivided this process into the following developmental stages as depicted in Figure 1A. Stage 1 is characterized by high proliferation and basement membrane deposition; stage 2 consists of a quiescent state and establishment of an outer cell layer with clear basolateral polarity; stage 3 consists of apoptosis of inner cells that allows formation of a hollow lumen in stage 4 . Here, we image single-cell ERK activity dynamics during different developmental stages of MCF10A acini formation. Stage 1 is characterized by high ERK frequency correlating with robust proliferation during initial acinus growth. This further correlates with rapid collective cell motility. During this stage, an abrupt transition to loss of collective cell motility correlates with lower ERK frequency. During stage 2, in which inner and outer cell subpopulations have been established, waves of single-cell ERK pulses across the acinus are observed. This correlates with domains of different ERK frequencies: inner cells display lower ERK frequency than outer cells, which correlates with apoptosis and lumen formation. Restoring high ERK frequency with a PIK3CA $\mathrm{H} 1047 \mathrm{R}$ mutation or through optogenetic control of ERK frequency, leads to survival of inner cells, hampering lumen formation. These results indicate how spatial control of ERK frequency and its ability to control apoptosis, survival and proliferation fates, enables mammary acinar morphogenesis.

\section{Results}

\section{Stage 1 proliferative acini exhibit spontaneous EGFR-dependent ERK pulses} whose frequency depends on collective cell migration speed.

To explore single-cell ERK activity dynamics during acinar morphogenesis, we created MCF10A reporter lines expressing the nuclear marker histone $2 \mathrm{~B}(\mathrm{H} 2 \mathrm{~B})$ fused to miRFP703 with either the ERK activity biosensor ERK KTR fused to mTurquoise2, or a geminin-mCherry S/G2/M cell cycle marker (Sakaue-Sawano et al., 2017). ERK KTR reports on ERK activity through reversible cytosolic shuttling that is induced by its phosphorylation by active ERK (Regot et al., 2014) (Figure S1A). These lines were then used to grow acini according to a classically-used protocol (Debnath et al., 2003). After a period of three days, in which EGF, serum and insulin were required for initial acinar growth (Figure S1B), these growth factors (GFs) were removed from the GFdepleted matrigel in order to study ERK signaling dynamics intrinsic to acinar morphogenesis. Using the geminin marker and a fluorogenic caspase substrate, we evaluated if our protocol recapitulated the proliferation, quiescence and apoptosis 
fates previously documented during the developmental stages that shape mature acini (Figure 1B-E) (Debnath et al., 2002; Liu et al., 2012). Stage 1 acini (imaged 4 days post-seeding) displayed elevated levels of proliferation and absence of apoptosis. Stage 2 and 3 acini (imaged 7 and 11 days post-seeding) revealed quiescent cells and increased apoptosis leading to lumen formation. Stage 4 acini (imaged 14 days postseeding) exhibited a mature lumen, displayed prominent apoptotic debris and a small increase in proliferation.

We then imaged single-cell ERK dynamics in stage 1 acini using 3D confocal spinning disk microscopy of both the H2B and ERK KTR channels with time resolutions of 3 5 minutes, until acini started to suffer from phototoxicity (which was observed after 10 - 23 hours of imaging). Visual inspection of micrographs of stage 1 acini revealed that different cells displayed asynchronous ERK pulses (Figure 2A) as previously observed in 2D cultures (Aikin et al., 2020; Albeck et al., 2013; Gagliardi et al., 2020). To quantify single-cell ERK activity trajectories, we used a custom-modified version of the opensource LEVER software (Wait et al., 2014; Winter et al., 2016) to segment and track nuclei based on their H2B signal; and to extract ERK activity trajectories by calculating the ratio of the ERK KTR intensities in the nucleus over a voxel mask around the nucleus (Figure 2B). Detrending of the ERK trajectories and normalization of the values to $[0,1]$ generated a reliable input for automated detection of ERK pulses (Figure 2B). Representative ERK trajectories revealed spontaneous ERK pulses with a wide range of amplitudes (Figure 2C). Trametinib-mediated MEK inhibition completely inhibited ERK activity, while gefitinib-mediated EGFR inhibition abolished ERK pulses (Figure 2D). Acute stimulation with $1 \mathrm{ng} / \mathrm{ml}$ EGF induced ERK pulses of similar amplitudes than spontaneous ones, while $100 \mathrm{ng} / \mathrm{ml}$ EGF induced ERK pulses of higher amplitudes (Figure 2E,F). These results show that stage 1 of acinar development is characterized by spontaneous, asynchronous, EGFR-dependent ERK pulses.

During stage 1, we observed a transition from a state of rapid collective motility, imposing a rotational movement on acini, to a state of slower motility leading to immobile acini (Figure 3A, Movie S1). This has previously been proposed to correlate with deposition of basement membrane (Wang et al., 2013). Strikingly, the reduction in migration speed associated with this transition correlated with a decrease in ERK frequency (Figure 3B, Figure S2A, Movie S2), without having a large effect on ERK pulse amplitude and duration (Figure S2B,C). 


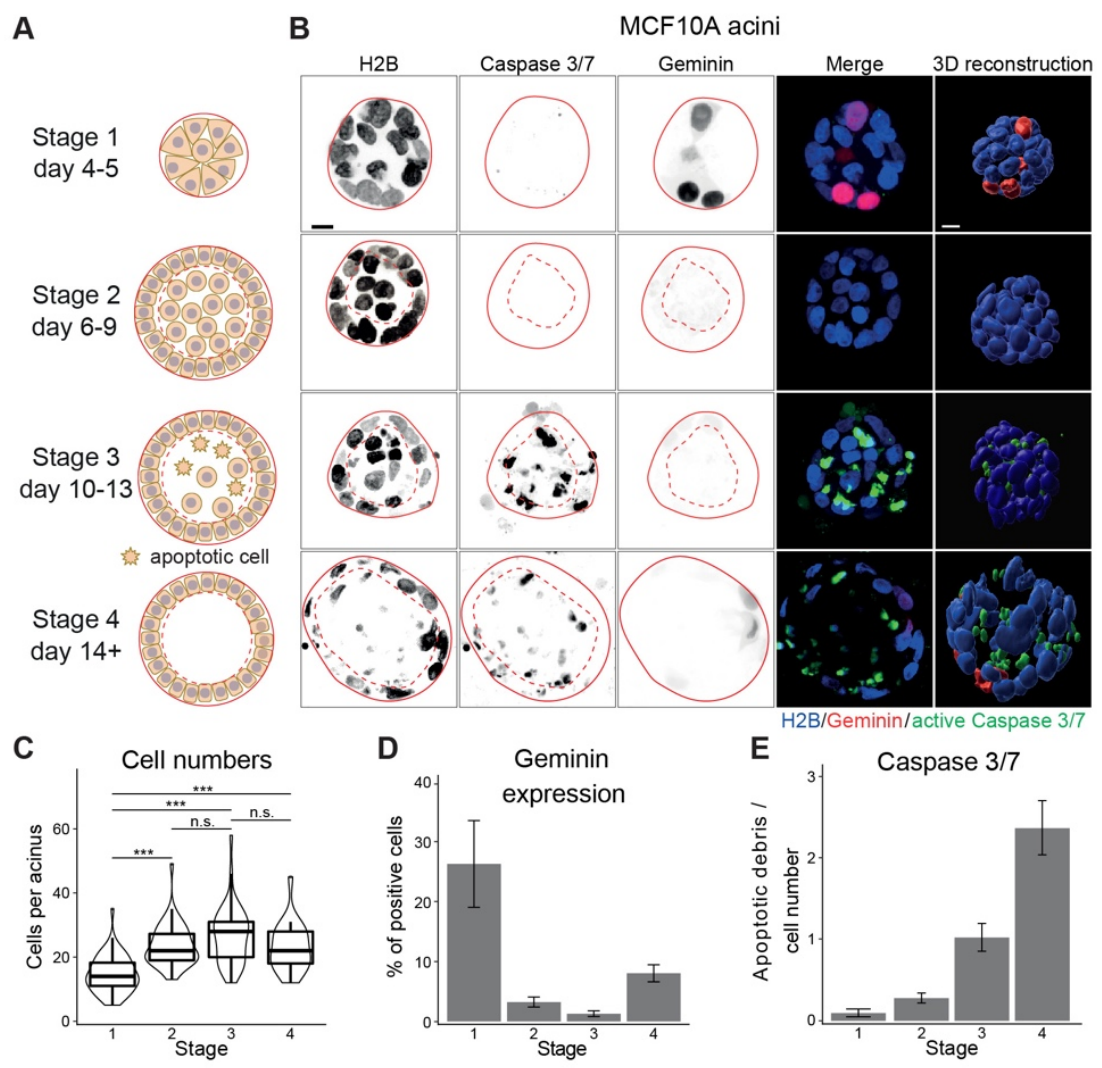

Figure 1 Morphogenesis of MCF10A acini can be divided in 4 distinct stages defined by different fate decisions. (A) Schematics of acinar developmental stages grown from single MCF10A cells. (B) Micrographs and 3D reconstructions of $\mathrm{H} 2 \mathrm{~B}$, caspase $3 / 7$ fluorogenic substrate and geminin signals in acini corresponding to the stages in (A). Micrographs show maximal intensity projections of equatorial $Z$ planes spanning $12 \mu \mathrm{m}$. Plain lines mark the borders of the acini, dashed lines mark the outer cell layer. Scale bar $=10 \mu \mathrm{m}$. (C) Cell numbers per acinus at different days corresponding to the 4 stages ( $N=28$ - 60 acini per condition). Box plots depict the median and the 25th and 75th percentiles, whiskers correspond to minimum and maximum non-outlier values. Significance values from Wilcoxon tests (n.s., P > 0.05; $\left.{ }^{* * *}, P<0.001\right)$. (D) Fraction of Geminin positive cells in the population at different days. Same acini as in $(C)$ were divided in 3 technical replicates per conditions with 8 - 20 acini each. (E) Number of Caspase 3/7 apoptotic debris divided by the acinar cell number at different days. Same acini as in (C). 
A

A Stage 1 acinus

$\triangle$ ERK on
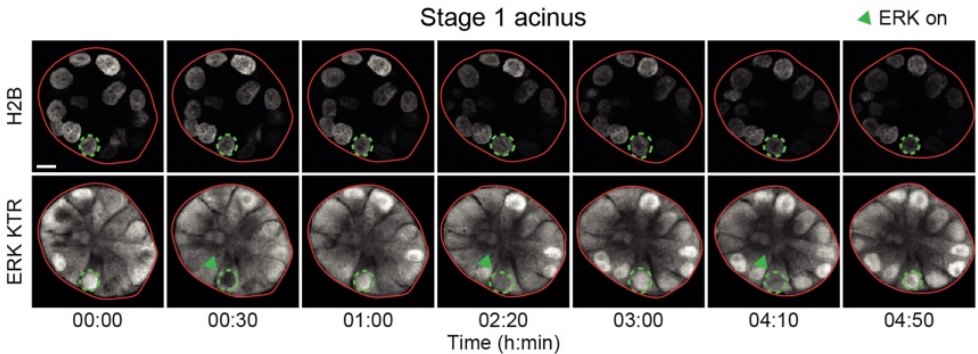

B

3D Nuclear segmentation

$\mathrm{H} 2 \mathrm{~B}$ rendering Segmentation Segmentation whole acinus whole acinus Inside view

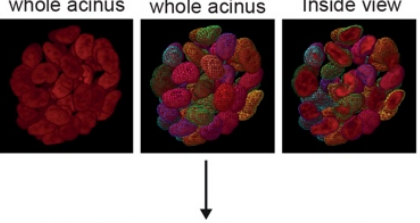

3D KTR cytosol / nucleus ratio

ERK KTR ERK KTR with Expansion of

Inside view segmentation nuclear mask
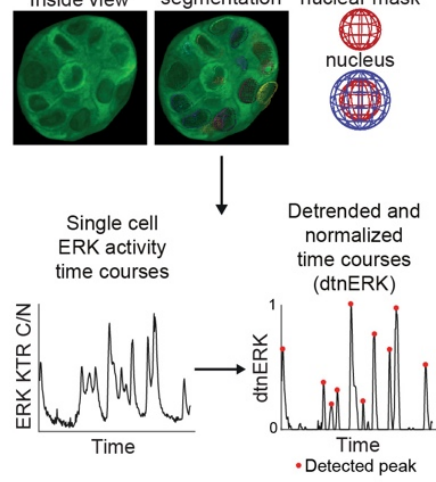

C

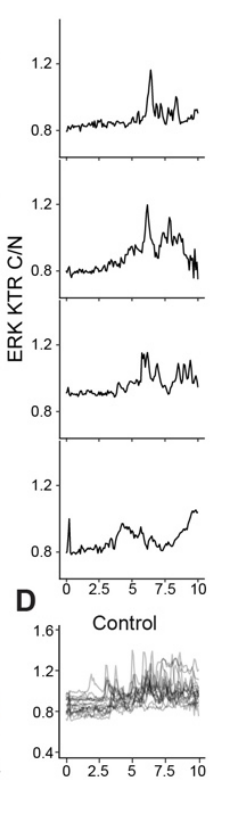

ERK activity

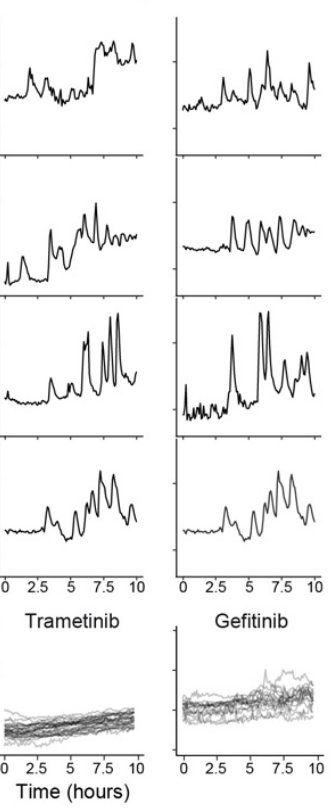

$\mathbf{E}$

ERK activity

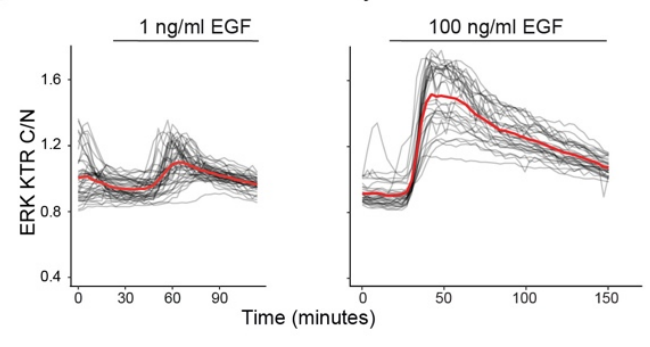

F ERK pulse amplitude

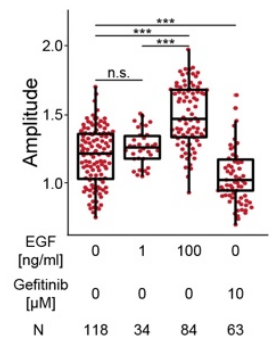

Figure 2 Developing acini exhibit spontaneous EGFR-dependent ERK pulses. (A) Time-series micrographs of a 5 days old acinus expressing fluorescent $H 2 B$ and $E R K$ KTR. The equatorial optical section is shown. Cells display spontaneous ERK activity pulses that can be detected by the nuclear to cytoplasmic translocation of ERK KTR. The cell indicated by a green dotted line exhibits robust ERK pulses, with active ERK being shown by an arrowhead. Red plain lines mark the borders of the acinus. Scale bar $=10 \mu \mathrm{m}$. (B) Image analysis pipeline to extract ERK trajectories from 3D time lapse datasets. Nuclei are segmented and tracked in 3D based on the H2B signal using LEVER. Single-cell ERK activity levels are calculated by dividing the median ERK KTR signal pixel intensities in the voxel mask around the nucleus by the one of the segmented nuclear volume. ERK pulses are detected on detrended ERK trajectories normalized to $[0,1]$ with 1 being the highest peak in the trajectory. $(\mathrm{C})$ Representative 
single-cell ERK trajectories from one acinus. (D) Overlayed ERK trajectories from control, gefitinib-treated and trametinib-treated acini. The control trajectories correspond to the same acinus as in (C). (E) ERK trajectories from acini treated with 1 or $100 \mathrm{ng} / \mathrm{ml}$ EGF at the indicated time points. Red lines represent the population averages. (F) ERK pulse amplitudes in cells from control, EGF-treated and gefitinibtreated acini. Box plots depict the median and the 25th and 75th percentiles, whiskers correspond to minimum and maximum non-outlier values. Wilcoxon tests (n.s., $\mathrm{P}>$ $\left.0.05 ;{ }^{* * *}, \mathrm{P}<0.001\right)$. 


\section{Stage 2 quiescent acini exhibit different ERK frequencies in inner and outer cells, which emerges from collective waves of ERK pulses.}

We then evaluated ERK dynamics in stage 2 quiescent acini that are larger, and are characterized by an outer layer of polarized cells, and a less organized inner cell mass destined for apoptosis during lumen formation at later stages (Debnath et al., 2002). When comparing stage 1 low motility and stage 2 acini, we observed a further reduction in single-cell ERK frequency (Figure 3D), while ERK pulse amplitudes and durations remained almost identical (Figure S2B,C). This change in ERK frequency resulted from a bimodal distribution in which a part of the cell population did not display ERK pulses anymore during the observation window (Figure 3D). Because ERK pulse frequency can regulate proliferation, survival and apoptosis fates in MCF10A cells (Aikin et al., 2020; Albeck et al., 2013; Gagliardi et al., 2020), and because inner cells in stage 2 acini are destined to undergo apoptosis, we evaluated ERK pulse frequencies in inner versus outer cells. Accordingly, we noticed a striking spatial pattern in which outer cells exhibited a significantly higher ERK frequency than inner cells, with the latter often not exhibiting ERK pulses at all during the observation window (Figure 4A,B). ERK activity amplitude and duration remained similar in inner and outer cells (Figure S3A,B). Together with our characterization of fate decisions (Figure 1), these results suggest the existence of a spatio-temporal mechanism that controls survival versus apoptosis fates through regulation of ERK frequency.

Another striking observation was that stage 2 acini exhibited spatially correlated ERK pulses in the form of waves that were spreading across multiple cells (Figure 4B-D, Movie S3). To quantify these spatial processes, we devised computational methods (Figure S3C-E, Materials and Methods) to detect, track, and extract a number of features that described ERK waves. These ERK waves were observed in all of the stage 2 acini that we imaged $(\mathrm{N}=11)$, and exhibited strikingly different geometries (Figure 4D). While some ERK pulses remained restricted to single cells, most of the ERK pulses occurred within collective waves (Figure 4E). ERK waves typically involved a median of 8 cells for a mean duration of 97 minutes (Figure 4F,G). However, a large variance was observed with some ERK waves involving as little as 2 and as much as 39 cells (almost the whole acinus). We observed that ERK waves, as well as isolated ERK pulses, were predominantly initiated in the outer versus the inner layer (Figure 4H). Further, ERK waves that originated in the outer layer displayed a higher 
A

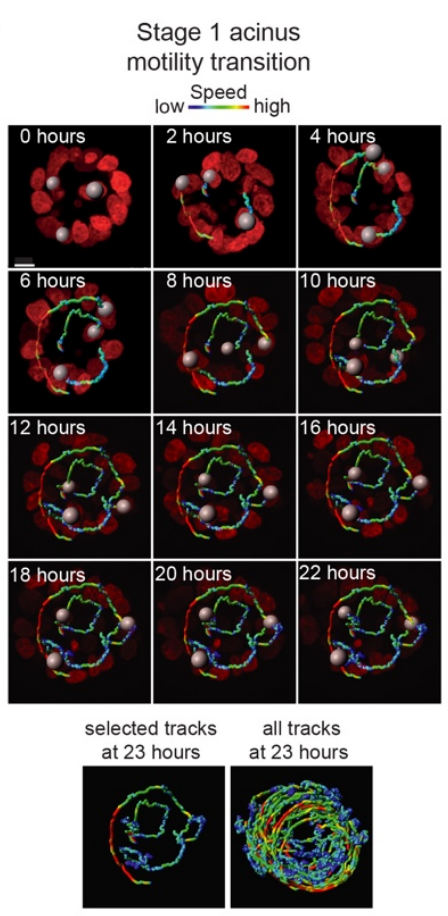

B

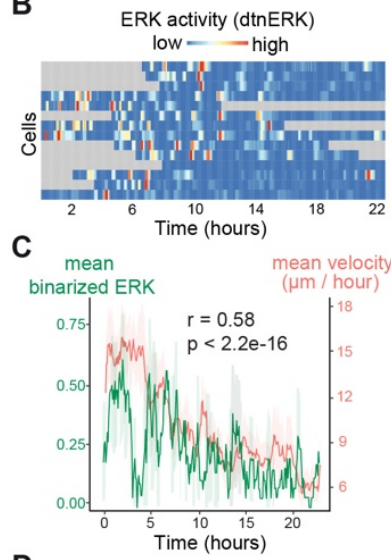

D

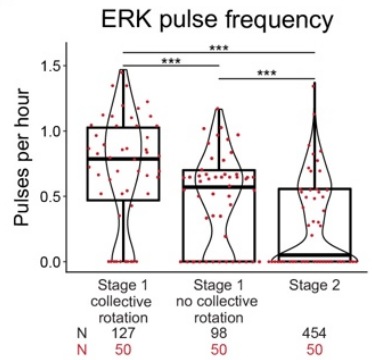

Figure 3 Stage 1 acini exhibit rapid collective cell migration and a high ERK pulse frequency that decreases upon transition to a low-motility stage $(A)$ Time series renderings of a cross section of an acinus transitioning from the rapid motility to the slow motility stage. Nuclei and motility tracks are shown (tracks are color-coded by instantaneous velocity). Scale bar $=10 \mu \mathrm{m}$. (B) Analysis of ERK activity in the acinus from (A). Heatmap shows detrended and normalized single cell ERK activity levels over time. Gray areas correspond to time points when a cell was not within the imaged volume. (C) Analysis of motility and ERK activity in the acinus from (A). Graph shows mean binarized ERK activity and mean instantaneous velocity with $95 \%$ confidence intervals of all imaged cells over time and their Pearson correlation coefficient. Mean binarized ERK activity is used as a measure for the fraction of the cell population in a state of active ERK. (D) ERK pulse frequency from trajectories at different developmental timepoints. Trajectories pooled from 7 (stage 1 rotation), 5 (stage 1 no rotation) and 11 (stage 2) acini. Box plots depict the median and the 25th and 75th percentiles, whiskers correspond to minimum and maximum non-outlier values. Dot plots show distribution of 50 randomly selected trajectories per condition. Significance values from Wilcoxon tests $\left({ }^{* * *}, \mathrm{P}<0.001\right)$. 
A

C ERK wave in stage 2 acinus ERK activity (dtnERK)

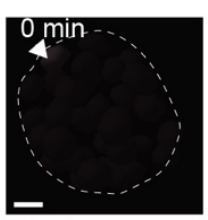
low high

B

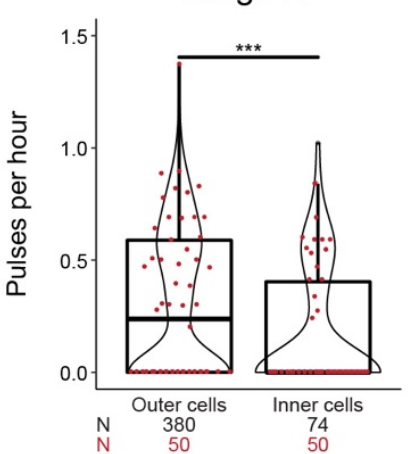

pulse frequency
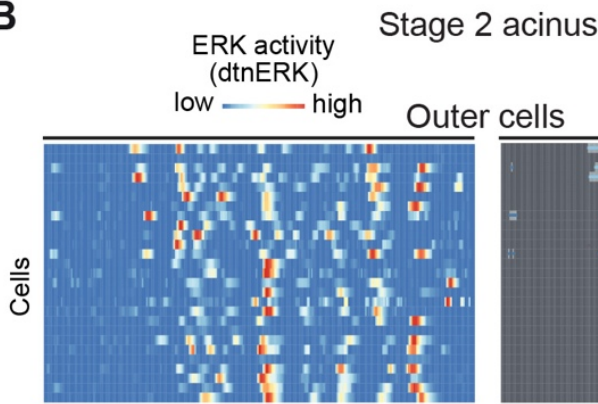

Inner cells

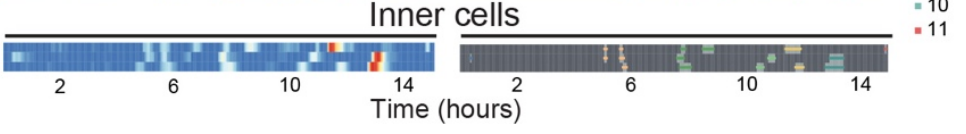

D $\quad$ ERK waves in different acini
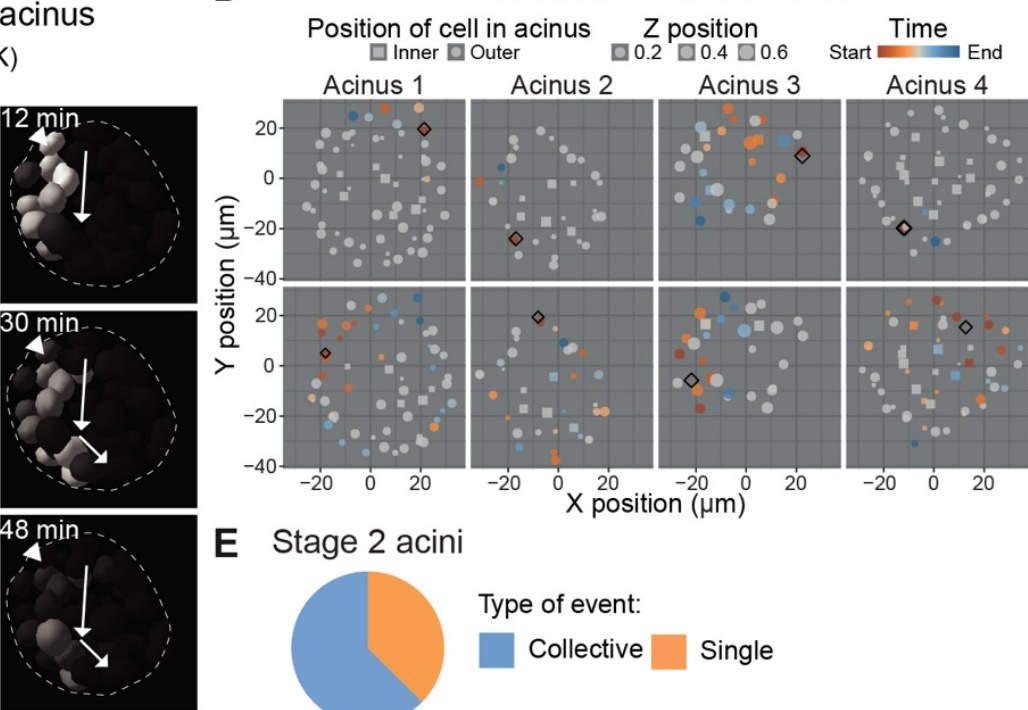

E Stage 2 acini

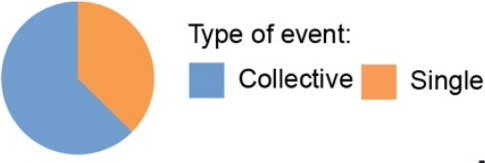

H

Frequency of single pulses and wave initiation per cell

Duration per wave

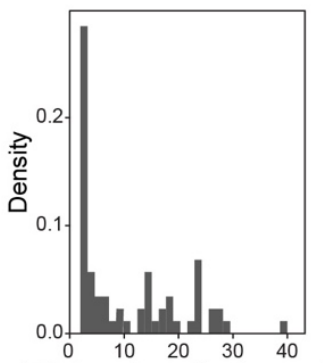

Total number of unique cells involved in a collective event

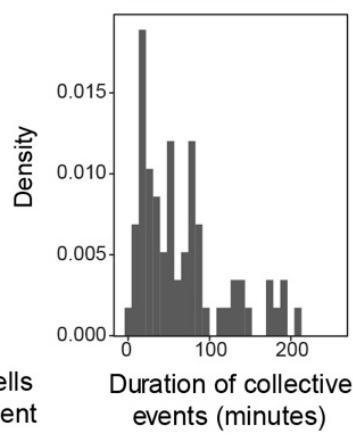

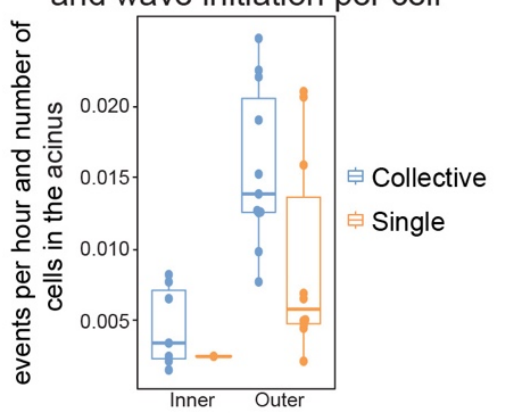

Position of the initiator cell
Penetration of waves

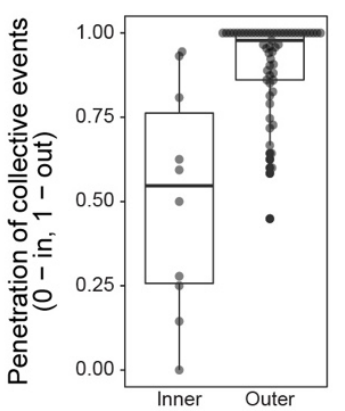

Position of the initiator cell

Figure 4 Stage 2 acini display different ERK pulse frequencies in inner and outer acini cell layers, and exhibit collective waves of ERK pulses. (A) ERK pulse frequency from trajectories of cells located in inner versus outer acini layers. Trajectories pooled from 11 acini. Box plots depict the median and the 25th and 75th percentiles, whiskers correspond to minimum and maximum non-outlier values. Dot plots show distribution of 50 randomly selected trajectories per condition. Wilcoxon test ( $\left.{ }^{* * *}, P<0.001\right)$. (B) Left panel: heatmap of detrended/normalized single-cell ERK trajectories in outer and inner cells of a representative acinus. Right panel: detection of individual ERK activity waves in the same acinus. (C) Representative time-serie micrographs of ERK wave ID 10 in (B). Nuclei are color-coded by ERK KTR ratios. 
Arrows depict wave directionality. The arrowhead indicates the initiator cell. Dashed line indicates the acinus border. Scale bar $=10 \mu \mathrm{m}$. (D) 2D projection representations of isolated ERK waves from four different acini. Size of cell position represents their relative $Z$ position and shape indicates if they belong to the inner or outer cell population. Cells that participate in the wave are color-coded by their relative time of activation. Top and bottom panels depict one isolated ERK wave for each acini. (E) Percentage of initiator events that remain restricted to a single cell vs those that lead to collective events longer than one frame. (F) Histogram of the total number of unique cells involved in individual collective events, that are longer than one frame and involve at least 2 cells. Box plots depict the median and the 25th and 75th percentiles, whiskers correspond to minimum and maximum non-outlier values. (G) Histogram of the duration of individual collective events in minutes. $(H)$ Average frequency of single and collective events per acinus, normalized by number of cells in the acinus. (I) Penetration of collective events across acini. Calculated as the time-averaged fraction of localisation of a collective event between the inner (0) and outer (1) cell layer. Box plots depict the median and the 25th and 75th percentiles, whiskers correspond to minimum and maximum non-outlier values. 
bias to remain at that location than those originating in inner cells (Figure 4I). We have previously shown that in 2D MCF10A cultures, apoptotic cells trigger ERK waves in their neighboring cells (Gagliardi et al., 2020). However, the ERK waves we observed here only rarely coincided with the onset of apoptotic events suggesting that they emerged from a different mechanism. Together, these results strongly suggest that ERK waves might contribute to spatially position different ERK pulse frequencies in inner and outer acinar cells.

\section{Oncogenic PIK3CA H1047R mutation correlates with increased ERK pulse frequency and loss of apoptosis-mediated lumen morphogenesis}

To explore the hypothesis that spatio-temporal control of ERK pulse frequency controls survival and apoptosis fates, we manipulated the latter to promote the survival fate in inner cells. In a screen of single-cell ERK dynamics in 2D monolayer cultures of MCF10A knockin lines expressing different oncogenic mutations, we found that cells harboring a breast cancer-associated point mutation PIK3CA H1047R (Figure $\mathrm{S} 4 \mathrm{~A}$ ) resulted in a higher ERK pulse frequency than their wild-type counterpart, while maintaining the same ERK pulse duration and amplitude (Figure 5A-D, S4B,C). PIK3CA encodes the alpha subunit of phospho-inositide-3 kinase (PI3K) and is frequently mutated in breast cancer (Cancer Genome Atlas Network, 2012). The higher ERK frequency is consistent with a population-average increase in ERK activity when measured using western blot (Gustin et al., 2009). At stage 1, rotating PIK3CA mutant acini displayed ERK pulse frequencies as high as those observed in their wildtype counterparts (Figure 5E). However, stage 1 non-rotating PIK3CA mutant acini did not display a striking decrease in ERK pulse frequency when compared to their wildtype counterparts (Figure 5E). In stage 2 acini, we observed a significant increase in ERK pulse frequency in PIK3CA mutant acini as compared to wild-type acini (Figure 5E,F, Movie S4). As for wild-type cells, PIK3CA mutant outer cells still displayed a higher ERK frequency than inner cells, leading the latter to exhibit a similar ERK pulse frequency as outer cells from wild-type acini. Treatment with gefitinib abolished spontaneous ERK pulses in PIK3CA mutant acini, indicating that PIK3CA-mediated modulation of ERK signaling might occur at the EGF receptor level (Figure S4F). We observed similar ERK waves as in wild-type cells (Figure 5F). Likely due to high heterogeneity in the ERK waves, we could not pinpoint a specific feature of ERK 
A

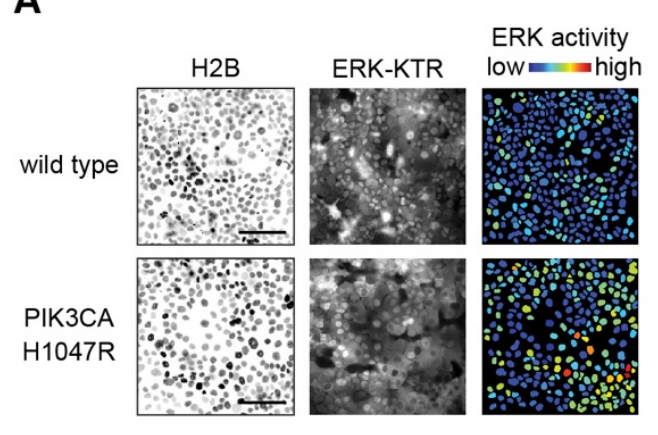

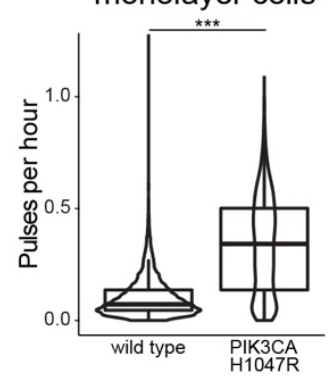

G
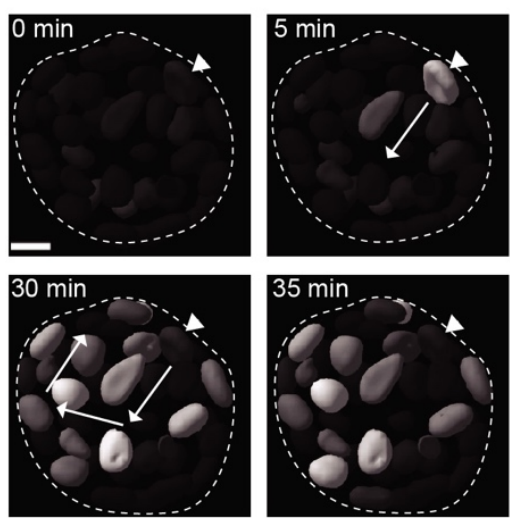

$\mathbf{E}$

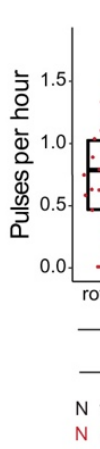

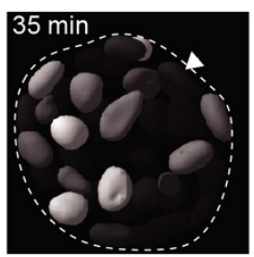

B

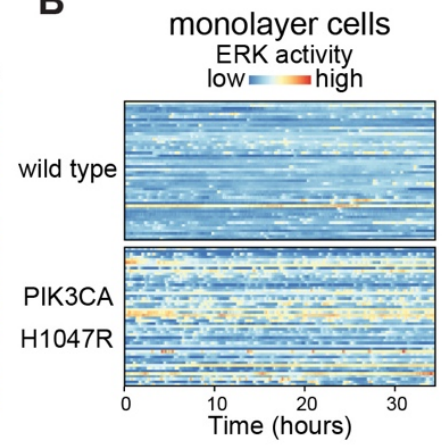

$\mathbf{F}$ ERK pulse frequency acini

C

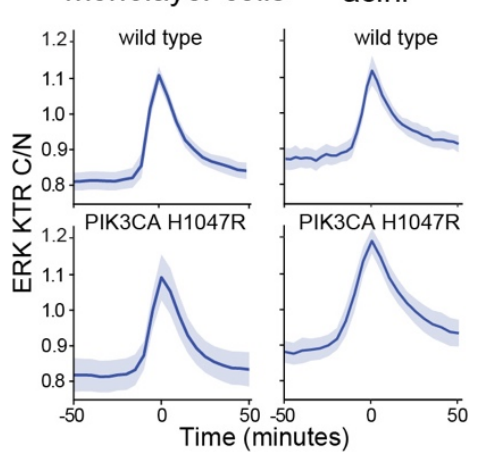

Stage 2 PIK3CA H1047R acinus ERK activity (dtnERK) low winh

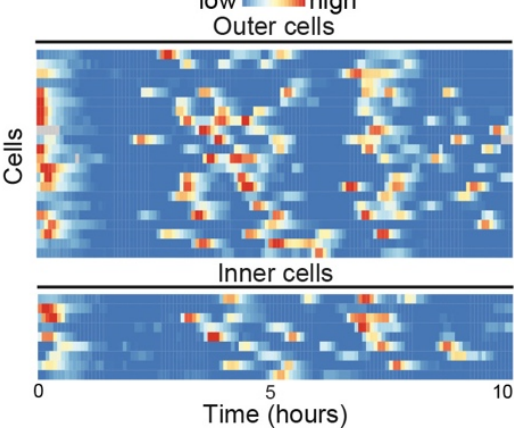

Stage 2 PIK3CA H1047R acinus ERK activity (dtnERK)

low high
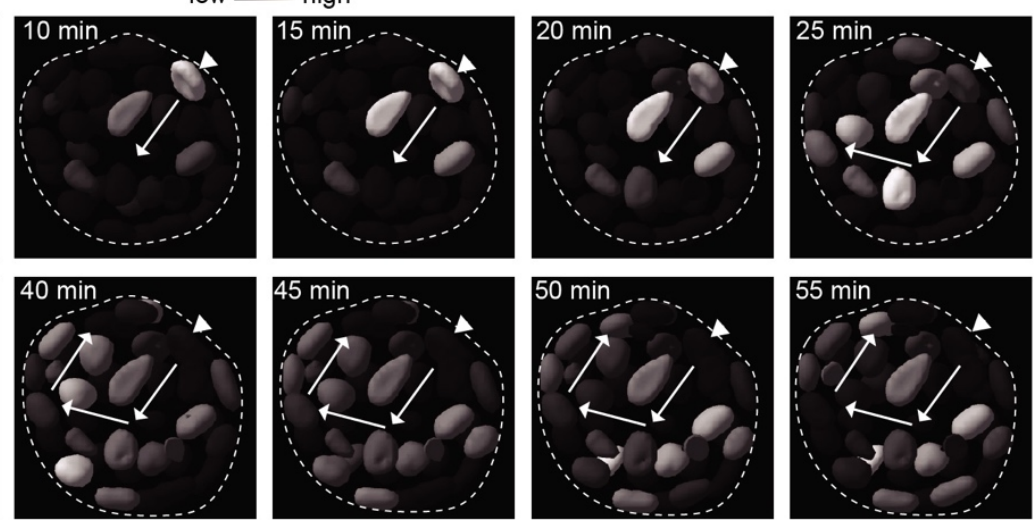

Figure 5 PIK3CA H1047R mutant acini exhibits higher ERK frequencies in 2D monolayer and 3D acini cultures. (A) Micrographs of wild-type and PIK3CA H1047R MCF10A 2D monolayers expressing fluorescent H2B (left panel) and ERK KTR (middle panel). Right panel: nuclei of the same cells color-coded by ERK KTR ratio. Scale bar $=100 \mu \mathrm{m}$. (B) Heatmap of single-cell ERK trajectories in wild-type and PIK3CA H1047R monolayers. (C) Average ERK trajectories from isolated pulses in wild-type and PIK3CA H1047R cells within monolayers (left panel) and acini (right panel). $95 \%$ confidence intervals are shown. Time $=0$ corresponds to maximal amplitude of peaks. (D) ERK frequencies in wild-type and PIK3CA H1047R monolayer cells. Box plots depict the median and the 25th and 75th percentiles, whiskers correspond to minimum and maximum non-outlier values. Wilcoxon test ${ }^{* * *}, \mathrm{P}<$ 0.001 ) (E) ERK frequencies of wild-type and PIK3CA H1047R cells at different stages and locations within the acinus. Mutant trajectories pooled from 7 (stage 1 rotation), 2 (stage 1 no rotation) and 6 (stage 2) acini. Wild-type data is the same as in figures 3 and 4 and shown again for comparison. Box plots depict the median and the 25 th and 
75th percentiles, whiskers correspond to minimum and maximum non-outlier values. Dot plots show distribution of up to 50 randomly selected trajectories per condition. Wilcoxon tests (n.s., $\mathrm{P}>0.05$; ${ }^{*}, \mathrm{P}<0.05,{ }^{* *}, \mathrm{P}<0.01$; ${ }^{* * *}, \mathrm{P}<0.001$ ). (F) Heatmap of detrended/normalized single-cell ERK trajectories in outer and inner cells of a representative stage 2 PIK3CA H1047R acinus. (G) Representative time-series of an ERK wave in a stage 2 PIK3CA H1047R acinus cross section. Nuclei are color-coded by ERK activity levels. Arrows show directionality of activation. The arrowhead indicates the initiator cell. Dashed line indicates the acinus border. Scale bar $=10 \mu \mathrm{m}$. 
waves that was accountable for the increased ERK frequency observed in PI3KCA mutant versus wild-type acini. These results indicate that mutant PIK3CA cells display an overall increased frequency of spontaneous ERK pulses that still can exhibit collective waves.

As already hinted in previous studies (Isakoff et al., 2005; Lauring et al., 2010), comparison of PIK3CA mutant versus wild-type acinar development revealed a marked increase in proliferation in mutant stage 1 acini as evidenced by augmented cell numbers, and geminin-positive cells (Figure 6A-E). Proliferation drastically diminished in stages 2 - 4, while remaining slightly higher than in wild-type acini, and displayed a small upshoot during stage 4 (Figure 6A,C). Inversely, in contrast to the steep apoptosis rise observed starting on day 7 in wild-type acini, apoptotic rates remained lower in PIK3CA mutant acini at all stages (Figure 6A,D). Consistent with these observations, cell numbers in PIK3CA mutant acini were always higher than in their wild-type counterparts, increased steadily until stage 2, slightly decreased at stage 3 , and displayed a small upshoot at stage 4 (Figure 6A,B). During stage 4, wildtype acini typically display cleared or partially cleared lumen. In contrast, all PI3KCA mutant acini failed to form a lumen (Figure 6E). These results indicate that an increase in proliferation at stage 1 , as well as strongly decreased apoptosis at stages 3 and 4 , enable the formation of acini with increased cell numbers and an absence of lumen in PIK3CA mutant cells. This correlates with the overall higher ERK frequency in PI3KCA versus wild-type acini.

\section{Optogenetic entrainment of MAPK signaling reveals ERK pulse frequency- dependent regulation of apoptosis-mediated lumen morphogenesis}

To further explore the role of ERK pulses as a frequency-encoded signal that controls apoptosis and survival fates required for lumen morphogenesis, we used optogenetics to evoke synthetic ERK pulses applied at different frequencies in the acinus. We used two optogenetic actuators to control ERK dynamics (Figure 7A): 1. optogenetic fibroblast growth factor receptor (optoFGFR) is a Cry2-based light-activatable receptor tyrosine kinase that activates ERK, Akt and calcium signaling (Kim et al., 2014). 2. optoRaf is a CIBN/Cry2-based system in which a truncated Raf form is recruited to a plasma membrane targeted anchor in a light-dependent fashion to specifically activate ERK (Aoki et al., 2017). We generated stable lines expressing any of the two optogenetic constructs, a spectrally compatible ERK KTR-mRuby, and H2B-miRFP. 


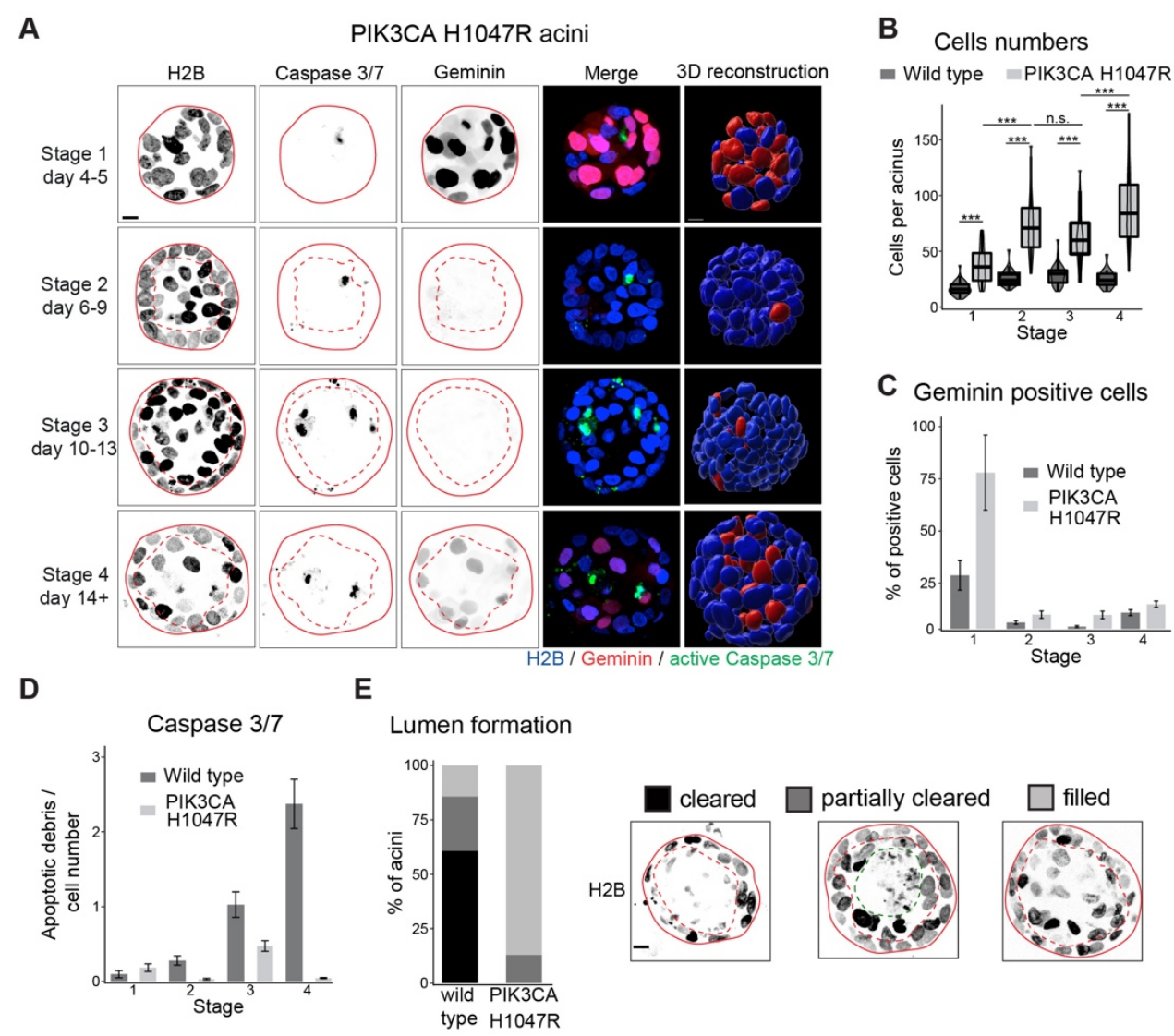

Figure 6 PIK3CA H1047R mutant acini exhibit increased proliferation, reduced apoptosis and absence of lumen formation. (A) Micrographs and 3D reconstructions of $\mathrm{H} 2 \mathrm{~B}$, caspase $3 / 7$ fluorogenic substrate and geminin signals in PIK3CA H1047R acini at different developmental stages. Micrographs show maximal intensity projections of equatorial $Z$ planes spanning $12 \mu \mathrm{m}$. Plain lines mark the acini borders, dashed lines mark the outer cell layer. Scale bar $=10 \mu \mathrm{m}$. (B) Cell numbers per acinus at different days corresponding to the 4 stages. $N=54-60$ PIK3CA $\mathrm{H} 1047 \mathrm{R}$ acini per day. Wild-type data in $(B)-(E)$ is the same as in figure 1 (shown here again for comparison with PIK3CA H1047R). Box plots depict the median and the 25th and 75th percentiles, whiskers correspond to minimum and maximum nonoutlier values. Wilcoxon tests (n.s., $\mathrm{P}>0.05$; ${ }^{* *}, \mathrm{P}<0.001$ ). (C) Fraction of Geminin positive cells in the population at different days. Same acini as in (B) divided in 3 technical replicates per day with $17-20$ acini each. (D) Numbers of Caspase 3/7 apoptotic debris divided by the acinar cell number at different days. Same acini as in (B) divided in 3 technical replicates per day with $17-20$ acini each. (E) Percentages of acini that either displayed a cleared, partially cleared or filled luminal space at day 14. Same acini as in (B) - (D) were used for the assessment. Representative examples used for classification are shown (maximal intensity projections of equatorial $Z$ planes spanning $12 \mu \mathrm{m}$ ). Plain lines mark acinar borders, red dashed lines mark the outer cell layer, the green dashed line marks the border between the cleared and filled part of the luminal space in the partially cleared condition. Scale bar $=10 \mu \mathrm{m}$. 
A

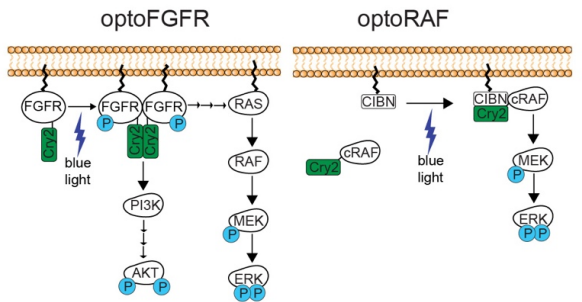

C

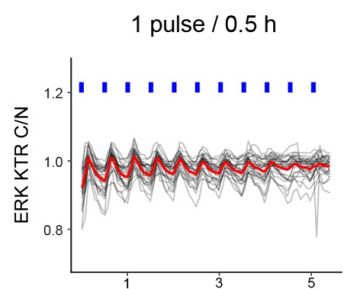

Optogenetic activation of optoFGFR in stage 2 acini
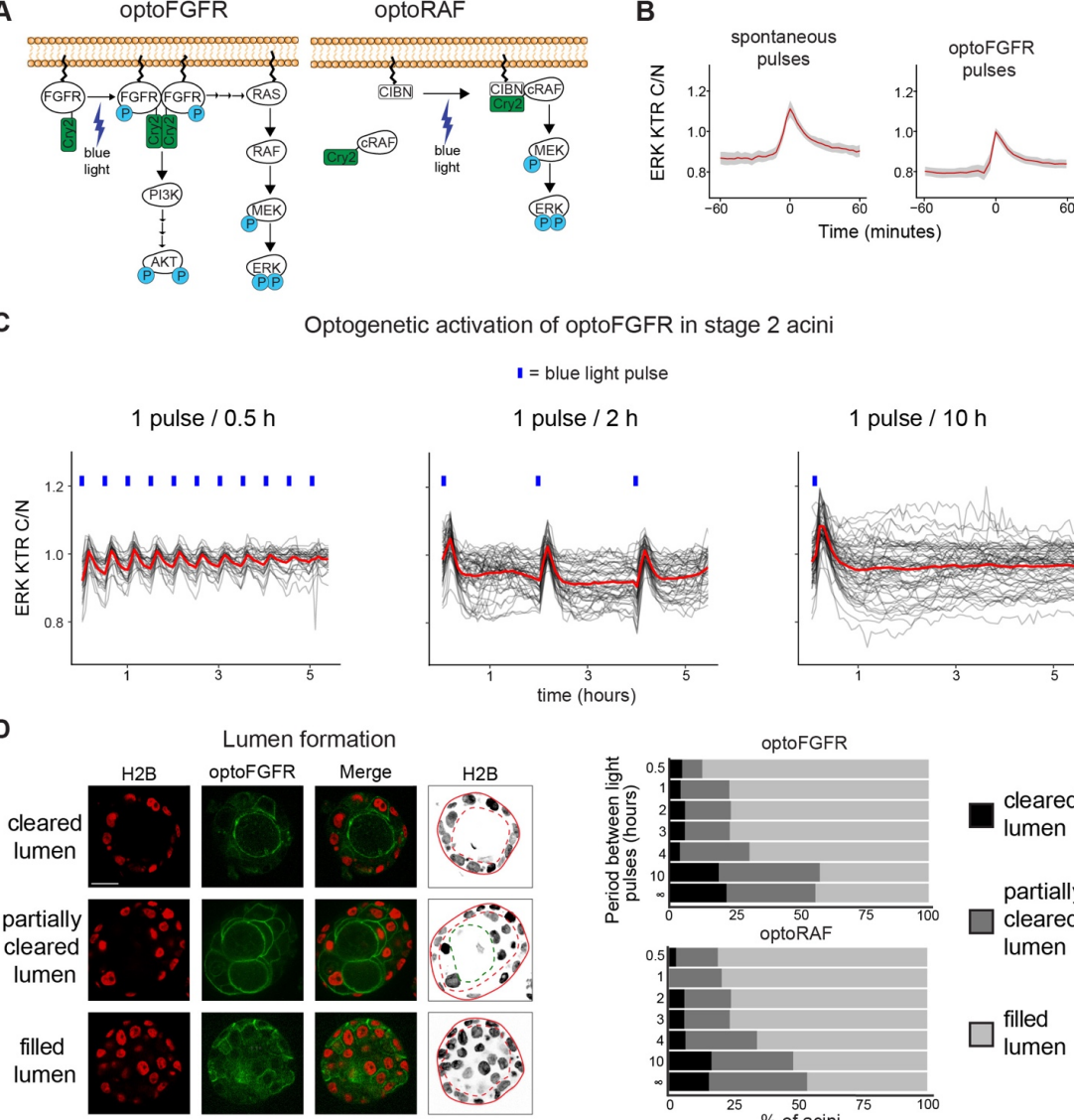

$\mathbf{I}=$ blue light pulse

1 pulse $/ 2 \mathrm{~h}$
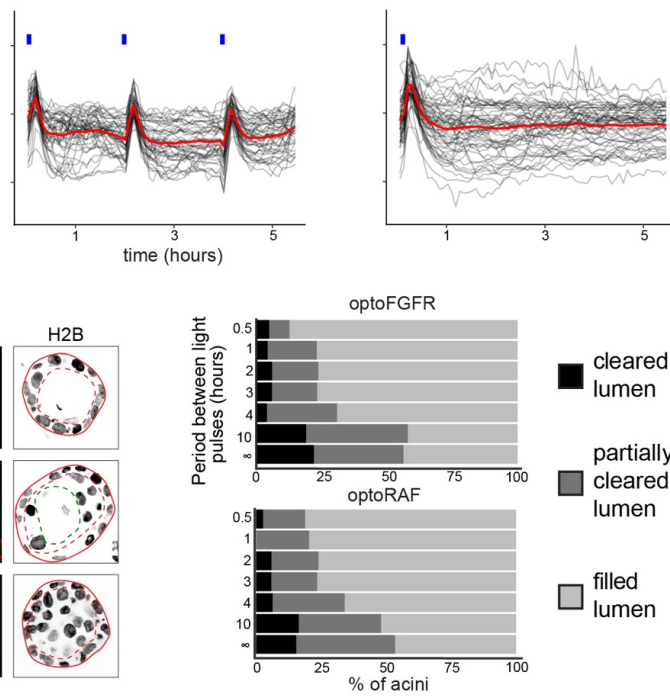

Figure 7 Optogenetically-entrained, synthetic frequency-modulated ERK pulse regimes control survival/apoptosis fate decisions (A) Cartoon of the optoFGFR and optoRAF systems. OptoFGFR consists of the intracellular domain of FGFR1 linked to the plasma membrane and a Cry2 PHR domain which dimerizes upon blue light stimulation, leading to receptor autophosphorylation and activation of downstream cascades. OptoRAF consists of a Cry2 linked to cRaf and a membranelinked $\mathrm{CIBN}$ domain. $\mathrm{CIBN}$ and $\mathrm{Cry} 2$ dimerize upon blue light stimulation which recruits cRaf to the plasma membrane where it phosphorylates Mek. (B) Average ERK trajectories from isolated spontaneous and optogenetically induced ERK pulses with $95 \%$ confidence interval. Time $=0$ corresponds to maximal amplitude of peaks. (C) Single cell ERK activity trajectories from 3 optoFGFR expressing acini stimulated with blue light pulses at different intervals under the microscope. (D) Percentages of optoFGFR and optoRAF-expressing acini that displayed cleared, partially cleared or filled luminal space at day 14, after they were kept for 7 days on an LED plate that emitted blue light pulses at defined intervals. $N=36-72$ acini per condition from 2 independent replicates. Representative examples used for classification are shown. Colored micrographs show single equatorial $Z$ planes. Black and white Micrographs show maximal intensity projections of equatorial $Z$ planes spanning $12 \mu \mathrm{m}$. Plain lines mark acini borders, red dashed lines mark the luminal space, the green dashed line marks the border between the cleared and filled part of the luminal space in the partially cleared condition. Scale bar $=20 \mu \mathrm{m}$. 
We focused on stage 2 acini in which inner cells start to display different ERK pulse frequencies than outer cells. We first evaluated the ability of blue light pulses delivered at different frequencies to evoke ERK activity pulses using our optogenetic actuators. We found that optoFGFR was able to evoke ERK pulses of similar amplitudes and durations as spontaneous pulses, at the specific frequencies at which light was applied. This allowed us to synchronize ERK dynamics in the entire acinus (Figure 7B,C). Because regulation of apoptosis/survival fates required for lumen formation during stages 3 and 4 occur on timescales of one week, we could not use our live cell imaging platform to study this process. We therefore used the multiwell plate illumination system LITOS (LED Illumination Tool for Optogenetic Stimulation) (Höhener et al., Manuscript in preparation) to entrain ERK dynamics at different frequencies in a tissue culture incubator (Figure S5A). We validated that this light stimulation modality was able to evoke similar ERK pulses as observed in our live cell imaging system (Figure S5B). Starting with day 7 after seeding acini kept in the dark, we stimulated stage 2 acini with light pulses delivered at different frequencies that correspond to those associated with survival or apoptosis fates for the next 7 days. We then scored the distribution of acini that exhibited filled lumen, partially cleared or cleared lumen (Figure 7D). Using both optoFGFR and optoRaf, we observed that ERK pulses induced every $0.5,1,2,3,4$ but not 10 pulses/hour led to survival of inner cells. This resulted in more acini with filled or partially cleared lumen. These results further strengthen the hypothesis that survival and apoptosis fates are regulated by a frequency-encoded ERK signal. The optoRaf results also suggest that ERK pulses are sufficient to induce the survival phenotype.

\section{Discussion}

Recent single-cell measurements of ERK activity dynamics in a variety of epithelial cells in $2 D$ cultures have revealed the existence of ERK pulses whose frequency can control proliferation (Albeck et al., 2013; Aoki et al., 2013), survival or apoptosis fates (Gagliardi et al., 2020; Valon et al., 2020). Such ERK pulses can also be spatiotemporally organised at the population level in the form of signaling waves that coordinate collective cell migration (Aoki et al., 2017; Hino et al., 2020), or extrusion of dying or cancer cells (Aikin et al., 2020; Gagliardi et al., 2020). Here we characterize single-cell ERK dynamics during 3D mammary acinar development. This involves a highly choreographed spatio-temporal coordination of fate decisions during different 
developmental episodes to initially grow a cell collective and organize it into a prototypical epithelial acinus with a lumen. A major challenge of our study was to correlate signaling dynamics that occur on timescales of minutes with proliferation, survival and apoptosis fate decisions that occur on timescales of days, during a developmental program that spans a period of two weeks. We therefore gated on specific developmental episodes that were accessible with our imaging photon budget. We observed that ERK pulses occurring in 2D epithelial culture systems also take place in 3D acini, indicating that the core signaling programs that shape ERK dynamics are independent from ECM dimensionality. These ERK pulses emerge from MAPK network properties such as signal amplification (leading to steep ERK activation), and negative feedback (leading to ERK adaptation) (Kholodenko et al., 2010). In acini cultured in GF-depleted matrigel, ERK pulses display heterogeneous amplitudes that are lower than those evoked by acute EGF stimulation (Figure 2C-F). This suggests that only very small inputs, such as minute amounts of proteolytically cleaved GFs, ECM interactions or mechanical forces, are sensed without leading to full amplitude ERK activation as observed with GF stimulation (Albeck et al., 2013; Aoki et al., 2017; Hino et al., 2020). However, we observed that the more complex spatio-temporal fate decision signaling occurring in acini, in comparison with 2D cultures, can be explained by relatively simple self-organization properties of ERK signaling. During stage 1, a reciprocal relationship between cell motility and ERK frequency, which is modulated by assembly of a basement membrane, might regulate the transition between proliferative and quiescent states. During stage 2, ERK waves might provide a spatiotemporal mechanism to specify two spatial domains with different ERK frequencies, leading to survival of outer cells and apoptosis in inner cells.

\section{Stage 1 Acini exhibit cell migration-dependent ERK frequency}

Stage 1 acini displayed either of the two different states: 1. a behaviour of rapid collective migration imposing a global rotation on the cell collective, with a transition to 2. a behaviour of slower, less coordinated cell motility (Figure $3 \mathrm{~A}$ ). This transition has been shown to result from assembly of an endogenous laminin-based basement membrane, in which rapid initial motility is in part required for this process (Wang et al., 2013). We observed that this transition in motility states correlated with a clear decrease in ERK frequency. We speculate that motility-dependent decrease of ERK frequency is part of the signaling program that allows the transition from proliferative 
stage 1 to quiescent stage 2 acini in which survival and apoptosis fates can then subsequently shape lumen formation. This is consistent with ERK frequency dependent regulation of proliferation, survival and apoptosis fates (Albeck et al., 2013; Gagliardi et al., 2020; Valon et al., 2020).

Previous studies have shown that inducing ERK activity increases motility through activation of myosin activity in MCF10A acini (Pearson and Hunter, 2007). This strongly suggests that ERK dynamics regulate both fate decisions and motility by the phosphorylation of different substrates in the nucleus and the cytosol respectively. Recently, an EGFR-dependent, mechanochemical feedback loop has been identified in epithelial cell collectives. ERK pulse-triggered myosin-based contractility of a leader cell mechanically activates an ERK pulse in a follower cell to reactivate contractility (Aoki et al., 2017; Boocock et al., 2020; Hino et al., 2020). This leads to the emergence of a positive feedback in which ERK pulses drive contractility/motility, and at the same time can reactivate ERK pulses in adjacent cells, leading to ERK waves that can coordinate collective motility. A similar mechanism might operate in stage 1 acini, however without the emergence of waves due to the very high ERK frequency that we observe. We speculate that assembly of a laminin basement membrane might modify the mechanochemical feedback loop,decreasing ERK pulsatility and motility. As a consequence, this might yield lower ERK pulse frequencies that allow the acini to transition to a quiescent state. Thus, the ability of a EGFR/ERK-based mechanochemical feedback loop to integrate and coordinate cytoskeletal dynamics with fate decision signaling might allow basement membrane formation to serve as a checkpoint between proliferative and quiescent states. Future experimental/modeling studies will be necessary to further refine this hypothesis.

\section{Stage 2 acini display ERK waves that specify different ERK frequencies in inner/outer cells}

In quiescent stage 2 acini, we observed a further shift to lower average ERK frequencies than observed in slowly migrating stage 1 acini (Figure 3D). This resulted from one subpopulation that displayed similar ERK frequencies as slowly migrating stage 1 acini, and another subpopulation that showed low frequency or absence of ERK pulses (Figure 3D). We then found that outer and inner cells, that were later destined to respectively survive and die by apoptosis, exhibited different ERK frequency distributions. Outer cells displayed ERK frequencies of typically one pulse 
every 2 hours on average, while inner cells pulsed rarer and often did not exhibit ERK pulses at all (Figure 4A). This is consistent with a similar phenomenon in serumstarved 2D MCF10A cultures in which dying cells evoke local waves of single-cell ERK pulses that each induce survival for approximately 4 hours (Gagliardi et al., 2020). Together, these results strongly suggest that medium and low ERK frequencies regulate survival and apoptosis fates respectively.

One important question is then how this spatio-temporal control of the ERK frequency pattern is achieved? Our characterization of ERK waves suggests that this might in part emerge from the traveling waves of single-cell ERK pulses taking place in stage 2 acini (Figure 4B-I). While isolated ERK pulses were also observed, most ERK pulses occurred within waves of various sizes (Figure 4D-G). The ERK waves most often originated in outer cells (Figure $4 \mathrm{H}$ ) which are mechanically linked through adherent junctions (Debnath et al., 2002). This potentially enables ERK activation through an EGFR mechanochemical crosstalk similar to the one already described above for collective migration (Aoki et al., 2017; Boocock et al., 2020; Hino et al., 2020). Consistently, ERK waves displayed a strong bias to propagate within the outer cell layer, and rarely propagated to inner cells (Figure 4I). Thus, given their limited range, ERK waves might act as a ruler for acinus size, allowing to specify different domains of ERK frequencies in inner and outer cells. This might ensure that apoptosis takes place only in inner cells in postmitotic acini that have grown large enough to develop a lumen, where ERK waves limit a high ERK frequency. Beyond this potential morphogenetic function, we speculate that stochastic ERK waves might also emerge in response to mechanical cues within the ECM, with the purpose of spatially finetuning fate decisions depending on possible mechanical fluctuations due to external perturbations. Future studies will be required to mechanistically explore how such ERK waves emerge and fine-tune acinar morphogenesis.

\section{Oncogenic PI3K signaling augments ERK pulse frequency in acini inner cells} and leads to loss of lumen formation by increasing survival fate decision.

We then sought to link the striking correlation we observed between spatio-temporal modulation of ERK pulse frequency and survival and apoptosis fates in stage 2 acini. The finding that knockin MCF10A 2D cultures harboring the clinically-relevant PIK3 H1047R mutation displayed elevated ERK pulse frequency, without affecting ERK pulse width or amplitude (Figure 5A-D, Figure S4B,C), provided an opportunity to 
explore a possible contribution of regulation of survival/apoptosis fates by ERK frequency. This mutated PI3KCA has been shown to induce increased proliferation, survival, and absence of lumen formation, both in vitro (Berglund et al., 2013; Chakrabarty et al., 2010; Chen et al., 2013; Isakoff et al., 2005; Lauring et al., 2010) and in vivo (Tikoo et al., 2012). Consistently, our high resolution characterization of fate decisions in PIK3CA H1047R acini revealed increased proliferation during stage 1 , followed by loss of proliferation but robust increase in survival during stages 2-4 (Figure 6A-E).

As in 2D cultures, PI3KCA mutant acini retained identical ERK pulse shapes as their wild-type counterparts (Figure 5C, Figure S4D,E). During stage 1, ERK frequencies were identical in wild-type and mutant acini. However, during stage 2, while still displaying a global decrease of ERK frequency in comparison with stage 1, PI3KCA mutant acini exhibited higher ERK pulse frequencies than their wild-type counterparts (Figure 5E). Mutant acini also retained the ability to produce ERK waves (Figure 5F). These results imply the existence of a crosstalk between oncogenic PI3K and MAPK signaling that has a subtle effect on spatio-temporal regulation of ERK frequency. Because ERK pulse shape is identical in wild-type and PI3KCA mutant cells, this crosstalk must occur upstream of the core Raf/MEK/ERK circuit with negative feedback that generates ERK pulses (Sparta et al., 2015). Consistently, gefitinibmediated EGFR inhibition abolished ERK pulses in both wild-type and PI3KCA mutant acini (Figure 1C,D, Figure S4F). Proteomics analysis of the mutant PI3KCA H1047R MCF10A knockin cells used in this study have revealed increased expression of the EGF-like ligand amphiregulin, and decreased expression of protein tyrosine phosphatase receptor type F (PTPRF) (Young et al., 2015). Secreted amphiregulin might lead to further EGFR stimulation (Sternlicht et al., 2005), while decreased PTPRF might lead to decreased EGFR inhibition (Du et al., 2013). Both molecular events might contribute to augmented EGFR activity, increasing the excitability of ERK pulses.

In essence, mutant PI3KCA acini display a subtle phenotype of globally increased ERK frequency, leading to its aberrant spatio-temporal regulation. However, formation of ERK waves and downregulation of ERK frequency in the transition from stage 1 to 2 are retained, which is consistent with stage-specific regulation of proliferation and survival fates (Figure 6). As a consequence, inner PI3KCA mutant cells display comparable ERK pulse frequencies to those observed in outer wild-type cells in stage 
2 acini, explaining the survival of the inner cells and absence of lumen formation. Indeed, given that $\mathrm{PI} 3 \mathrm{~K}$ signaling itself can regulate proliferation/survival fates (Manning and Cantley, 2007), we cannot exclude that additional inputs contribute to the control of these fate decisions. However, the striking parallel between the regulation of ERK frequency and fate decisions in both wild-type and PI3KCA mutant acini strongly suggests that spatio-temporal regulation of ERK frequency is a major determinant in controlling fate decisions.

\section{Optogenetic entrainment of ERK frequency regulates survival versus apoptosis}

\section{fates}

Finally, as an additional method to explore the role of ERK frequency in fate decisions, we used optogenetics to evoke synthetic, synchronous ERK pulse frequencies in the entire acini. Using optoFGFR that activates ERK, Akt and Calcium signaling (Kim et al., 2014), and optoRaf that solely activates ERK (Aoki et al., 2017), we found that synthetic ERK pulse regimes applied at frequencies higher than one pulse every 10 hours led to loss of inner cell apoptosis, and loss of lumen formation (Figure 7D). This worked for optoFGFR that activates both ERK and Akt signaling, as well as for optoRaf that solely activates ERK. This supports the concept that ERK frequency specifically is responsible for spatio-temporal control of cell fates.

In summary, we provide initial insight into how single-cell ERK dynamics are spatiotemporally regulated to control different fate decisions in space and time during the morphogenesis of a simple prototype organ structure. The spatial control of ERK pulse frequency-dependent fates is based on different modalities of dynamic selforganisation properties of MAPK signaling in cellular communities. Future studies are required to mechanistically understand how these dynamic signaling states are encoded and spatially organised, and if they provide robustness against environmental perturbations occurring during development. This will require the ever expanding arsenal of optogenetic tools to manipulate specific single cells and evaluate how the cell collective responds. Further questions include how additional signaling pathways might fine tune this morphogenetic process, and how the ERK frequency is decoded into transcriptional programs that actuate the different fates that shape acinus morphogenesis. 


\section{Material and methods}

\section{D cell culture}

MCF10A cells were cultured in DMEM/F12 supplemented with $5 \%$ horse serum, 20 $\mathrm{ng} / \mathrm{ml}$ recombinant human EGF (Peprotech), $0.5 \mathrm{mg} / \mathrm{ml}$ hydrocortisone (SigmaAldrich/Merck), $10 \mu \mathrm{g} / \mathrm{ml}$ insulin (Sigma-Aldrich/Merck), $200 \mathrm{U} / \mathrm{ml}$ penicillin and 200 $\mu \mathrm{g} / \mathrm{ml}$ streptomycin. The PIK3CA H1047R knockin cell line (Gustin et al., 2009) was a gift of Ben Ho Park. To generate stable cell lines, cells were transfected with FuGene (Promega) according to the manufacturer's protocol and clones were selected by antibiotic resistance and image-based screening.

\section{D cell culture}

For acinus formation, single MCF10A cell suspensions were mixed with 4 volumes of growth factor - reduced Matrigel (Corning) at $4{ }^{\circ} \mathrm{C}$ and spread evenly on the surface of glass bottom cell culture plates at a concentration of $1.5 \times 10^{4} \mathrm{cells} / \mathrm{cm}^{2}$. Acini were cultured in DMEM/F12 supplemented with $2 \%$ horse serum, $20 \mathrm{ng} / \mathrm{ml}$ recombinant human EGF, $0.5 \mathrm{mg} / \mathrm{ml}$ hydrocortisone, $10 \mu \mathrm{g} / \mathrm{ml}$ insulin, $200 \mathrm{U} / \mathrm{ml}$ penicillin and 200 $\mu \mathrm{g} / \mathrm{ml}$ streptomycin. Horse serum, insulin and EGF were removed after 3 days in culture. For live imaging, $25 \mathrm{mM}$ Hepes was added to the medium prior to mounting on the microscope. CellEvent Caspase 3/7 Green Detection Reagent was obtained from Thermo Fisher Scientific and used according to the manufacturer's protocol.

\section{Plasmids}

ERK KTR-mTurquoise2 and ERK KTR-mRuby2 were generated by fusion of the coding region of ERK KTR (Regot et al., 2014) with that of mTurquoise2 (Goedhart et al., 2012) or mRuby2 (Lam et al., 2012). H2B-miRFP703 was generated by fusion of the coding region of human $\mathrm{H} 2 \mathrm{~B}$ clustered histone 11 (H2BC11) with that of miRFP703 (Shcherbakova et al., 2016). Geminin-mCherry was generated by fusion of the ubiquitylation domain of human Geminin (Sakaue-Sawano et al., 2017) to mCherry. The above mentioned fusion proteins were cloned in the piggyBac vectors pMP-PB, pSB-HPB (Balasubramanian et al., 2016) (gift of David Hacker, EPFL), or pPB3.0.Blast, an improved version of pPB generated in our lab.

pPB3.0-PuroCRY2-cRAF-mCitrine-P2A-CIBN-KrasCT, referred to in the manuscript as OptoRAF, was generated in the following way: The CRY2-cRaf sequence was 
excised from pCX4puro-CRY2-cRAF (gift from Kazuhiro Aoki, (Aoki et al., 2017)) using EcoRI and Notl. mCitrine was PCR amplified from the optoFGFR plasmid, while adding Notl and Xhol sites, and digested. Both sequences were ligated into pPB3.0Puro, previously digested with EcoRI and Xhol. The GSGP2A-CIBN-KRasCT sequence (synthesized by GENEWIZ) was digested with BsrGI and AfIII and ligated into pPB3.0-Puro-CRY2-cRAF-mCitrine.

The piggyBac plasmids were co-transfected with a helper plasmid expressing a hyperactive piggyBac transposase (Yusa et al., 2011).

Lyn-cytoFGFR1-PHR-mCit, expressing myristoylated FGFR1 cytoplasmic region fused with the PHR domain of cryptochrome2 and mCitrine (gift from Won Do Heo (Addgene plasmid \# 59776), (Kim et al., 2014)), referred to in the manuscript as OptoFGFR, was subcloned in a lentiviral backbone for stable cell line generation.

\section{Imaging}

All acini images were acquired on an epifluorescence Eclipse Ti2 inverted fluorescence microscope (Nikon) equipped with a CSU-W1 spinning disk confocal system (Yokogawa) and a Plan Apo VC 60X water immersion objective (NA = 1.2). For time lapse imaging, laser-based autofocus was used. Images were acquired with a Prime 95B or a Prime BSI sCMOS camera (both Teledyne Photometrics) at 16 bit depth. Temperature, $\mathrm{CO} 2$ and humidity were controlled throughout live imaging with a temperature control system and gas mixer (both Life Imaging Services).

All monolayer cell images were acquired on an epifluorescence Eclipse Ti inverted fluorescence microscope (Nikon) with a Plan Apo 20x air objective (NA =0.8) or a Plan Apo 40X air objective (NA = 0.9). Laser-based autofocus was used throughout imaging. Images were acquired with an Andor Zyla 4.2 plus camera at 16 bit depth.

Both microscopes were controlled by NIS elements (Nikon).

\section{Drug experiments}

Gefitinib was obtained from Sigma-Aldrich/Merck and added to the acini 2 hours before imaging at a concentration of $10 \mu \mathrm{M}$. Trametinib was obtained from Selleck Chemicals and added to the acini during imaging at a concentration of $5 \mu \mathrm{M}$.

\section{Optogenetic experiments}


For short term optogenetics experiments performed directly on the microscope, acini were illuminated with wide field blue light (470 nm LED) at defined time points during spinning disc time lapse imaging. Acini expressing optoFGFR were illuminated for 10 ms at $50 \%$ LED intensity. The NIS elements JOBS module was used to program the imaging and stimulation patterns.

For long term optogenetic stimulation with the LITOS system, glass bottom 96-well cell culture plates with 7-day old acini were fitted on a 32 x 64 RGB LED matrix (Boxtec) inside a cell culture incubator. The matrix was connected to a custom printed circuit board with an ESP32 microcontroller. This system was programmed to emit 1minute blue light pulses at maximal intensity at defined intervals for 7 days, after which lumen formation efficiency was assessed. Acini were fixed with $4 \%$ paraformaldehyde prior to imaging.

\section{Image analysis of 3D acini}

The open source LEVER software (Cohen, 2014; Wait et al., 2014; Winter et al., 2016) was used to analyze the 3D time lapse movies. The LEVER software was updated to include improved processing and visualization capabilities. The processing pipeline began with a GPU-accelerated 3D non-local means denoising algorithm (Wait et al., 2019). After denoising, a new ensemble-based segmentation algorithm was applied. This ensemble segmentation combined an adaptive thresholding into foreground/background regions with an anisotropic 3-D Laplacian of Gaussian filter targeted to a specific cell radius to separate touching cells (Winter et al., 2019). The base segmentation was run at different cell radii and the results were combined using unsupervised learning techniques from the field of algorithmic information theory (Cohen et al., 2009). Here, the radii evaluated ranged from $2.5 \mu \mathrm{m}$ to $4 \mu \mathrm{m}$ in $0.5 \mu \mathrm{m}$ steps. These values were set empirically based on expected cell size ranges. Following segmentation, the cells were tracked using Multitemporal Association Tracking (Winter et al., 2011; Winter et al., 2012).

Following segmentation and tracking of the image sequences, the ERK KTR signal was extracted and processed to a detrended and normalized signal. To extract the ERK KTR signal, distance transforms were computed for each segmented image. The interior distance transform assigned each cell interior voxel a numeric value indicating 
its distance starting at the cell boundary and increasing to the centroid. The exterior distance transform assigned each boundary voxel a numeric value indicating its distance to the nearest cell-assigned voxel. The exterior distance transform also provided the identity of the nearest cell-assigned voxel for each background voxel. The ERK KTR signal was computed as the ratio of the image values around the center of the cell to the image values around the boundary of the cell. The center

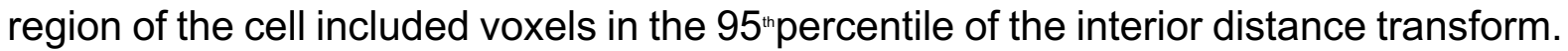
For the boundary region of the cell we included interior voxels within one unit of the boundary and exterior voxels within three units of the boundary. The resulting ERK KTR signal was computed as the ratio of the median voxel value in the outer region to the median voxel value in the inner region. The extracted ERK KTR signals for each cell had different base intensities and showed different amounts of fluctuation. To normalize this and to allow for quantitative comparison and visual representation with different cell ERK KTR expression levels we computed a detrended and normalized ERK KTR signal as follows. The signal $S$ was first detrended by subtracting the median filter signal, $S_{d}=S$-median_filter $(S)$. The signal was then normalized to the range $[0,1]$ using $S_{n d}=S_{d} / \max \left(S_{d}\right)$, unless the signal range in the detrended trajectory was below an empirically set threshold. Inner and outer cells in stage 2 acini were identified visually based on the 3D reconstructions in LEVER.

For segmentation and quantification of steady state Z-stacks, CellProfiler 3.1.8 (McQuin et al., 2018) with 3D functionalities was used. Nuclei were identified based on H2B-miRFP signals. Apoptotic debris were identified using Caspase 3/7 Green Detection kit signals using adaptive thresholding and watershed segmentation. Geminin-mCherry intensities were measured within nuclei voxel masks. ERK KTR cytosolic/nuclear intensity ratios were generated by measuring median ERK KTR intensities in the nuclear area and in a spherical voxel mask 1 pixel around the nuclear objects. Imaris software (Bitplane) was used for 3D rendering of confocal stacks and to track and measure motility parameters in Figure 3.

\section{Image analysis of 2D cell cultures}

Nuclei of monolayer cells were segmented using llastik (Berg et al., 2019) and CellProfiler 3.0. Ilastik was used for training a random forest classifier on different pixel features of the H2B-miRFP channel and background pixels. The training data was 
generated by manual annotation of 20 - 50 cells. The resulting probability map was imported into CellProfiler for thresholding and segmentation. Cytosolic ERK KTR fluorescence intensities were measured by expansion of the nuclear objects. Cells were tracked using $\mu$-track 2.2.1 (Jaqaman et al., 2008).

\section{Data analysis}

All analysis and visualization of ERK activity peaks and time series was performed with custom R/Matlab/Python code.

\section{ERK activity pulse detection}

ERK activity peaks were identified and counted by the following steps. 1. Application of a short median filter to smooth the time series. 2. Application of a long median filter to produce a bias estimate which was subtracted from the smoothed time series. 3 . Detrended time-series with real peaks were then identified by selecting those with an activity difference above an empirical threshold. Those were rescaled to $[0,1]$ and a local maxima detection algorithm was used to identify peaks above an amplitude of 0.1 .

\section{Identification of collective events}

To identify waves of collective ERK activation we developed a custom code and implemented it in $\mathrm{R}$. The algorithm works on a binarised signal that is calculated by detrending and normalising ERK KTR cytosolic/nuclear intensity ratio time series as described above (Figure S3C,D). The algorithm searches for the first occurrence of cells with ERK switched on (Figure S3E). If several such cells exist and they are located within a threshold radius, they initiate the first collective event. A single active cell can also become a collective event. In the next time point, the algorithm repeats the search for active cells and compares their distances to cells in the previous frame. If new active cells are located within the threshold distance to active cells at the previous time point, they are added to respective collective events. If new active cells are located outside of the threshold distance, they form new collective events. This process of growing clusters of collective activity is repeated for all remaining time points. The resulting statistics include the total number of cells involved in a collective 
event, the duration and the average size of an event and the location (inner or outer layer) of the cells that initiates the event.

\section{Data and code availability}

The open-source code for LEVERJS is available at https://leverjs.net/git. All 3D datasets that were analyzed with LEVERJS for this publication can be browsed interactively at https://leverjs.net/mcf10a_3d.

\section{Acknowledgments}

The authors are grateful to Ben Ho Park for providing the MCF10A PIK3CA H1047R knockin MCF10A cells, to Kazuhiro Aoki for the optoRaf constructs, and to Won Do Heo for the optoFGFR constructs. This work was supported by grants from the Swiss National Science Foundation and the Swiss Cancer League to Olivier Pertz and from a Human Frontiers Science Program grant to Olivier Pertz and Andrew Cohen. We acknowledge support of the Microscopy Imaging Center of the University of Bern (https://www.mic.unibe.ch/).

\section{Author contribution}

$P E, P A G$ and $O P$ designed the study, PE and PAG performed experiments and analyzed data, $M D$ and M-AJ analyzed data, CD provided expertise with the optogenetic tools, TH provided expertise with the optogenetic LED plate, ARC performed image analysis using LEVERJS, PE and OP wrote the paper.

\section{Conflict of interest}

The authors declare that they have no conflict of interest

\section{References}

Aikin, T.J., Peterson, A.F., Pokrass, M.J., Clark, H.R., and Regot, S. (2020). MAPK activity dynamics regulate non-cell autonomous effects of oncogene expression. Elife 9.

Albeck, J.G., Mills, G.B., and Brugge, J.S. (2013). Frequency-modulated pulses of ERK activity transmit quantitative proliferation signals. Mol. Cell 49, 249-261. 
Anderson, L.R., Sutherland, R.L., and Butt, A.J. (2010). BAG-1 overexpression attenuates luminal apoptosis in MCF-10A mammary epithelial cells through enhanced RAF-1 activation. Oncogene 29, 527-538.

Aoki, K., Kumagai, Y., Sakurai, A., Komatsu, N., Fujita, Y., Shionyu, C., and Matsuda, M. (2013). Stochastic ERK activation induced by noise and cell-to-cell propagation regulates cell density-dependent proliferation. Mol. Cell 52, 529-540.

Aoki, K., Kondo, Y., Naoki, H., Hiratsuka, T., Itoh, R.E., and Matsuda, M. (2017). Propagating wave of ERK activation orients collective cell migration. Dev. Cell 43, 305-317.e5.

Balasubramanian, S., Wurm, F.M., and Hacker, D.L. (2016). Multigene expression in stable $\mathrm{CHO}$ cell pools generated with the piggyBac transposon system. Biotechnol. Prog. 32, 1308-1317.

Berglund, F.M., Weerasinghe, N.R., Davidson, L., Lim, J.C., Eickholt, B.J., and Leslie, N.R. (2013). Disruption of epithelial architecture caused by loss of PTEN or by oncogenic mutant p110a/PIK3CA but not by HER2 or mutant AKT1. Oncogene 32, 4417-4426.

Berg, S., Kutra, D., Kroeger, T., Straehle, C.N., Kausler, B.X., Haubold, C., Schiegg, M., Ales, J., Beier, T., Rudy, M., et al. (2019). ilastik: interactive machine learning for (bio)image analysis. Nat. Methods 16, 1226-1232.

Boocock, D., Hino, N., Ruzickova, N., Hirashima, T., and Hannezo, E. (2020). Theory of mechanochemical patterning and optimal migration in cell monolayers. Nat. Phys.

Cancer Genome Atlas Network (2012). Comprehensive molecular portraits of human breast tumours. Nature 490,61-70.

Chakrabarty, A., Rexer, B.N., Wang, S.E., Cook, R.S., Engelman, J.A., and Arteaga, C.L. (2010). H1047R phosphatidylinositol 3-kinase mutant enhances HER2mediated transformation by heregulin production and activation of HER3. Oncogene 29, 5193-5203.

Chen, N., Eritja, N., Lock, R., and Debnath, J. (2013). Autophagy restricts proliferation driven by oncogenic phosphatidylinositol 3-kinase in three-dimensional culture. Oncogene 32, 2543-2554. 
Ciarloni, L., Mallepell, S., and Brisken, C. (2007). Amphiregulin is an essential mediator of estrogen receptor alpha function in mammary gland development. Proc Natl Acad Sci USA 104, 5455-5460.

Cohen, A.R. (2014). Extracting meaning from biological imaging data. Mol. Biol. Cell $25,3470-3473$.

Cohen, A.R., Bjornsson, C.S., Temple, S., Banker, G., and Roysam, B. (2009). Automatic summarization of changes in biological image sequences using algorithmic information theory. IEEE Trans. Pattern Anal. Mach. Intell. 31, 13861403.

Debnath, J., Mills, K.R., Collins, N.L., Reginato, M.J., Muthuswamy, S.K., and Brugge, J.S. (2002). The role of apoptosis in creating and maintaining luminal space within normal and oncogene-expressing mammary acini. Cell 111, 29-40.

Debnath, J., Muthuswamy, S.K., and Brugge, J.S. (2003). Morphogenesis and oncogenesis of MCF-10A mammary epithelial acini grown in three-dimensional basement membrane cultures. Methods 30, 256-268.

Du, W.W., Fang, L., Li, M., Yang, X., Liang, Y., Peng, C., Qian, W., O’Malley, Y.Q., Askeland, R.W., Sugg, S.L., et al. (2013). MicroRNA miR-24 enhances tumor invasion and metastasis by targeting PTPN9 and PTPRF to promote EGF signaling. J. Cell Sci. 126, 1440-1453.

Fata, J.E., Mori, H., Ewald, A.J., Zhang, H., Yao, E., Werb, Z., and Bissell, M.J. (2007). The MAPK(ERK-1,2) pathway integrates distinct and antagonistic signals from TGFalpha and FGF7 in morphogenesis of mouse mammary epithelium. Dev. Biol. 306, 193-207.

Finlay, D., Healy, V., Furlong, F., O'Connell, F.C., Keon, N.K., and Martin, F. (2000). MAP kinase pathway signalling is essential for extracellular matrix determined mammary epithelial cell survival. Cell Death Differ. 7, 302-313.

Gagliardi, P.A., Dobrzynski, M., Jacques, M.-A., Dessauges, C., Hughes, R.M., and Pertz, O. (2020). Collective ERK/Akt activity waves orchestrate epithelial homeostasis by driving apoptosis-induced survival. BioRxiv.

Goedhart, J., von Stetten, D., Noirclerc-Savoye, M., Lelimousin, M., Joosen, L., Hink, M.A., van Weeren, L., Gadella, T.W.J., and Royant, A. (2012). Structure-guided 
evolution of cyan fluorescent proteins towards a quantum yield of $93 \%$. Nat. Commun. 3, 751.

Gustin, J.P., Karakas, B., Weiss, M.B., Abukhdeir, A.M., Lauring, J., Garay, J.P., Cosgrove, D., Tamaki, A., Konishi, H., Konishi, Y., et al. (2009). Knockin of mutant PIK3CA activates multiple oncogenic pathways. Proc Natl Acad Sci USA 106, 28352840.

Hino, N., Rossetti, L., Marín-Llauradó, A., Aoki, K., Trepat, X., Matsuda, M., and Hirashima, T. (2020). ERK-Mediated Mechanochemical Waves Direct Collective Cell Polarization. Dev. Cell 53, 646-660.e8.

Huebner, R.J., Neumann, N.M., and Ewald, A.J. (2016). Mammary epithelial tubes elongate through MAPK-dependent coordination of cell migration. Development 143, 983-993.

Inman, J.L., Robertson, C., Mott, J.D., and Bissell, M.J. (2015). Mammary gland development: cell fate specification, stem cells and the microenvironment.

Development 142, 1028-1042.

Isakoff, S.J., Engelman, J.A., Irie, H.Y., Luo, J., Brachmann, S.M., Pearline, R.V., Cantley, L.C., and Brugge, J.S. (2005). Breast cancer-associated PIK3CA mutations are oncogenic in mammary epithelial cells. Cancer Res. 65, 10992-11000.

Jaqaman, K., Loerke, D., Mettlen, M., Kuwata, H., Grinstein, S., Schmid, S.L., and Danuser, G. (2008). Robust single-particle tracking in live-cell time-lapse sequences. Nat. Methods 5, 695-702.

Kholodenko, B.N., Hancock, J.F., and Kolch, W. (2010). Signalling ballet in space and time. Nat. Rev. Mol. Cell Biol. 11, 414-426.

Kim, N., Kim, J.M., Lee, M., Kim, C.Y., Chang, K.-Y., and Heo, W.D. (2014).

Spatiotemporal control of fibroblast growth factor receptor signals by blue light. Chem. Biol. 21, 903-912.

Lam, A.J., St-Pierre, F., Gong, Y., Marshall, J.D., Cranfill, P.J., Baird, M.A., McKeown, M.R., Wiedenmann, J., Davidson, M.W., Schnitzer, M.J., et al. (2012). Improving FRET dynamic range with bright green and red fluorescent proteins. Nat. Methods 9, 1005-1012. 
Lauring, J., Cosgrove, D.P., Fontana, S., Gustin, J.P., Konishi, H., Abukhdeir, A.M., Garay, J.P., Mohseni, M., Wang, G.M., Higgins, M.J., et al. (2010). Knock in of the AKT1 E17K mutation in human breast epithelial cells does not recapitulate oncogenic PIK3CA mutations. Oncogene 29, 2337-2345.

Lavoie, H., Gagnon, J., and Therrien, M. (2020). ERK signalling: a master regulator of cell behaviour, life and fate. Nat. Rev. Mol. Cell Biol.

Liu, J.S., Farlow, J.T., Paulson, A.K., Labarge, M.A., and Gartner, Z.J. (2012). Programmed cell-to-cell variability in Ras activity triggers emergent behaviors during mammary epithelial morphogenesis. Cell Rep. 2, 1461-1470.

Manning, B.D., and Cantley, L.C. (2007). AKT/PKB signaling: navigating downstream. Cell 129, 1261-1274.

McQuin, C., Goodman, A., Chernyshev, V., Kamentsky, L., Cimini, B.A., Karhohs, K.W., Doan, M., Ding, L., Rafelski, S.M., Thirstrup, D., et al. (2018). CellProfiler 3.0: Next-generation image processing for biology. PLoS Biol. 16, e2005970.

Paine, I.S., and Lewis, M.T. (2017). The Terminal End Bud: the Little Engine that Could. J. Mammary Gland Biol. Neoplasia 22, 93-108.

Pearson, G.W., and Hunter, T. (2007). Real-time imaging reveals that noninvasive mammary epithelial acini can contain motile cells. J. Cell Biol. 179, 1555-1567.

Reginato, M.J., Mills, K.R., Becker, E.B.E., Lynch, D.K., Bonni, A., Muthuswamy, S.K., and Brugge, J.S. (2005). Bim regulation of lumen formation in cultured mammary epithelial acini is targeted by oncogenes. Mol. Cell. Biol. 25, 4591-4601. Regot, S., Hughey, J.J., Bajar, B.T., Carrasco, S., and Covert, M.W. (2014). Highsensitivity measurements of multiple kinase activities in live single cells. Cell 157, 1724-1734.

Sakaue-Sawano, A., Yo, M., Komatsu, N., Hiratsuka, T., Kogure, T., Hoshida, T., Goshima, N., Matsuda, M., Miyoshi, H., and Miyawaki, A. (2017). Genetically encoded tools for optical dissection of the mammalian cell cycle. Mol. Cell 68, 626640.e5.

Sebastian, J., Richards, R.G., Walker, M.P., Wiesen, J.F., Werb, Z., Derynck, R., Hom, Y.K., Cunha, G.R., and DiAugustine, R.P. (1998). Activation and function of 
the epidermal growth factor receptor and erbB-2 during mammary gland morphogenesis. Cell Growth Differ. 9, 777-785.

Shcherbakova, D.M., Baloban, M., Emelyanov, A.V., Brenowitz, M., Guo, P., and Verkhusha, V.V. (2016). Bright monomeric near-infrared fluorescent proteins as tags and biosensors for multiscale imaging. Nat. Commun. 7, 12405.

Sparta, B., Pargett, M., Minguet, M., Distor, K., Bell, G., and Albeck, J.G. (2015). Receptor Level Mechanisms Are Required for Epidermal Growth Factor (EGF)stimulated Extracellular Signal-regulated Kinase (ERK) Activity Pulses. J. Biol. Chem. 290, 24784-24792.

Sternlicht, M.D., Sunnarborg, S.W., Kouros-Mehr, H., Yu, Y., Lee, D.C., and Werb, Z. (2005). Mammary ductal morphogenesis requires paracrine activation of stromal EGFR via ADAM17-dependent shedding of epithelial amphiregulin. Development 132, 3923-3933.

Tikoo, A., Roh, V., Montgomery, K.G., Ivetac, I., Waring, P., Pelzer, R., Hare, L., Shackleton, M., Humbert, P., and Phillips, W.A. (2012). Physiological levels of Pik3ca(H1047R) mutation in the mouse mammary gland results in ductal hyperplasia and formation of ERa-positive tumors. PLoS ONE 7, e36924.

Valon, L., Levillayer, F., Davidovič, A., Chouly, M., Cerqueira-Campos, F., and Levayer, R. (2020). Robustness of epithelial sealing is an emerging property of local ERK feedbacks driven by cell elimination. BioRxiv.

Wait, E., Winter, M., Bjornsson, C., Kokovay, E., Wang, Y., Goderie, S., Temple, S., and Cohen, A.R. (2014). Visualization and correction of automated segmentation, tracking and lineaging from 5-D stem cell image sequences. BMC Bioinformatics 15, 328.

Wait, E., Winter, M., and Cohen, A.R. (2019). Hydra image processor: 5-D GPU image analysis library with MATLAB and python wrappers. Bioinformatics 35, 53935395.

Wang, H., Lacoche, S., Huang, L., Xue, B., and Muthuswamy, S.K. (2013). Rotational motion during three-dimensional morphogenesis of mammary epithelial acini relates to laminin matrix assembly. Proc Natl Acad Sci USA 110, 163-168. 
Winter, M.R., Fang, C., Banker, G., Roysam, B., and Cohen, A.R. (2012). Axonal transport analysis using Multitemporal Association Tracking. Int. J. Comput. Biol. Drug Des. 5, 35-48.

Winter, M., Wait, E., Roysam, B., Goderie, S.K., Ali, R.A.N., Kokovay, E., Temple, S., and Cohen, A.R. (2011). Vertebrate neural stem cell segmentation, tracking and lineaging with validation and editing. Nat. Protoc. 6, 1942-1952.

Winter, M., Mankowski, W., Wait, E., Temple, S., and Cohen, A.R. (2016). LEVER: software tools for segmentation, tracking and lineaging of proliferating cells.

Bioinformatics 32, 3530-3531.

Winter, M., Mankowski, W., Wait, E., De La Hoz, E.C., Aguinaldo, A., and Cohen, A.R. (2019). Separating touching cells using pixel replicated elliptical shape models. IEEE Trans. Med. Imaging 38, 883-893.

Young, C.D., Zimmerman, L.J., Hoshino, D., Formisano, L., Hanker, A.B., Gatza, M.L., Morrison, M.M., Moore, P.D., Whitwell, C.A., Dave, B., et al. (2015). Activating PIK3CA Mutations Induce an Epidermal Growth Factor Receptor (EGFR)/Extracellular Signal-regulated Kinase (ERK) Paracrine Signaling Axis in Basal-like Breast Cancer. Mol. Cell. Proteomics 14, 1959-1976.

Yusa, K., Zhou, L., Li, M.A., Bradley, A., and Craig, N.L. (2011). A hyperactive piggyBac transposase for mammalian applications. Proc Natl Acad Sci USA 108, 1531-1536. 